\title{
The bodily illusion in adverse conditions: virtual arm ownership during visuo-motor mismatch
}

Barbara Caola ${ }^{1}$, Martina Montalti ${ }^{1}$, Alessandro Zanini $^{1}$, Antony Leadbetter $^{1}$ and Matteo Martini ${ }^{1}$

${ }^{1}$ University of East London, School of Psychology, Stratford campus, Water Lane, London, UK

Keywords: body ownership, virtual reality, multisensory integration, illusory movement, rubber hand illusion,

Corresponding author:

Matteo Martini, $\mathrm{PhD}$

School of Psychology

University of East London (UEL)

Stratford campus

Water Lane

London

E15 4LZ

E-mail: M.Martini@uel.ac.uk 


\begin{abstract}
Classically, body ownership illusions are triggered by cross-modal synchronous stimulations, and hampered by multisensory inconsistencies. Nonetheless, the boundaries of such illusions have been proven to be highly plastic. In this immersive virtual reality study we explored whether it is possible to induce a sense of body ownership over a virtual body part during visuo-motor inconsistencies, with or without the aid of concomitant visuo-tactile stimulations. From a first person perspective participants watched a virtual tube moving or an avatar's arm moving, with or without concomitant synchronous visuo-tactile stimulations on their hand. Three different virtual arm/tube speeds were also investigated, while all participants kept their real arms still. The subjective reports show that, synchronous visuotactile stimulations effectively counteract the effect of visuo-motor inconsistencies, but at slow arm movements a feeling of body ownership might be successfully induced even without concomitant multisensory correspondences. Possible therapeutical implications of these findings are discussed.
\end{abstract}




\section{Introduction}

An accurate representation of what belongs to and forms our bodies and what doesn't, is essential to achieve a fully functioning normal life. Body ownership in particular, is a perceptual status that is deemed to be fundamental for self-consciousness (Tsakiris, Carpenter, James, \& Fotopoulou, 2010). An impairment at this level yields dramatic consequences which are evident in asomatognosic and somatoparaphrenic patients, that report a delusion of disownership of their own body parts (Berlucchi \& Aglioti, 2010; Feinberg, Venneri, Simone, Fan, \& Northoff, 2010; Vallar \& Ronchi, 2009). The sense of body ownership is processed in specific brain areas, including the premotor cortices (Ehrsson, Spence, \& Passingham, 2004), and derives from the integration of multisensory information such as visual, tactile and proprioceptive input (Makin, Holmes, \& Ehrsson, 2008; Petkova, Khoshnevis, \& Ehrsson, 2011). However, even in healthy subjects, this representation is not steady but it can be altered quite easily. Many experimental findings in fact show that a body ownership illusion (BOI) over fake body parts can be induced in a few seconds (Ehrsson, Holmes, \& Passingham, 2005; Ehrsson, Spence, \& Passingham, 2004; Kalckert \& Ehrsson, 2012) and it is possible extend the experience of ownership to an entire artificial body (Slater, Spanlang, Sanchez-Vives, \& Blanke, 2010). One well-known example is the rubber hand illusion (RHI) where the tactile feedback produced by stroking the hidden real hand is coupled to the sight of a prosthetic hand stroked at the same time. This type of stimulation eventually leads to the feeling that the prosthetic hand belongs to one's body (Botvinick \& Cohen, 1998). This illusion can be successfully extended to mannequin and virtual full bodies (Petkova \& Ehrsson, 2008; Slater et al., 2010).

Although it is accepted that an anatomical correspondence between the real and the dummy body part must be respected in order for the illusion of ownership to occur (Tsakiris \& Haggard, 2005), a growing body of evidence is showing the impressive flexibility of BOIs. 
We now know, for instance, that it is possible to experience ownership over arms that appear much longer than normal (Kilteni, Normand, Sanchez-Vives, \& Slater, 2012), that look injured or particularly hairy (Osumi, Imai, Ueta, Nobusako, \& Morioka, 2014), that dynamically change their skin color (Martini, Perez-Marcos, \& Sanchez-Vives, 2013), that are semi-transparent (Martini, Kilteni, Maselli, \& Sanchez-Vives, 2015) or which are completely invisible (Guterstam, Gentile, \& Ehrsson, 2013). Similarly, BOIs can be experienced over invisible bodies (Guterstam, Abdulkarim, \& Ehrsson, 2015), dolls' and giants' bodies (van der Hoort, Guterstam, \& Ehrsson, 2011), children bodies (Banakou, Groten, \& Slater, 2013), bodies that resemble famous characters (Osimo, Pizarro, Spanlang, \& Slater, 2015) or that belong to other racial groups (Peck, Seinfeld, Aglioti, \& Slater, 2013). Importantly, BOIs are not only useful to investigate what cognitive factors influence body representation and to unravel the neural underpinnings behind it, but they also contribute to shedding light on what is the relationship between the perception of our body and our behaviour. Thanks to the studies focusing on this topic, it is currently known that the feeling of ownership over a different body or body part can have dramatic effects on subsequent perceptual, emotional, behavioural and physiological responses, according to the physical properties of the body that is object of the illusion (Banakou et al., 2013; Bergström, Kilteni, \& Slater, 2016; Haggard \& Jundi, 2009; Ma, Sellaro, Lippelt, \& Hommel, 2016; Maister et al., 2015; Preston \& Ehrsson, 2016; van der Hoort \& Ehrsson, 2014). Therefore, revealing under which conditions BOIs can occur is essential for understanding the mechanisms that influence self-representation and consequently how this representation, in turn, may influence perception.

Classically, studies making use of BOIs rely on synchronous visuo-tactile (or visuo-motor) stimulation to induce such experience, based on the assumption that intermodal matching is the 'conditio sine qua non' to induce self-attribution. Nonetheless, it has been recently shown 
that just the passive observation of the fake body, as long as it seen from a first person perspective (1PP) and it is co-located with the real body, is sufficient to trigger the illusion of body ownership (Martini, Kilteni, Maselli, \& Sanchez-Vives, 2015; Maselli \& Slater, 2013; Tieri, Tidoni, Pavone, \& Aglioti, 2015). This feeling of body ownership over an external body part by mere visual capture has been also reported in hemiplegic patients with and without disturbed sensation of limb ownership (Martinaud, Besharati, Jenkinson, \& Fotopoulou, 2017). Yet the majority of studies making use of BOI paradigms relies on synchronous multimodal stimulation. Recently it has been shown how, when visuo-tactile stimulation is used, a slow tactile stimulation which targets the tactile $\mathrm{C}$ fibers leads to a stronger BOI (Crucianelli, Krahé, Jenkinson, \& Fotopoulou, 2017). Moreover, an asynchronous visuo-tactile (or visuo-motor) stimulation is considered to be the essential characteristic of the typical control condition, that is where no BOI is expected to take place. However, despite the intermodal mismatch, such condition does not always bring about significantly lower sense of body ownership compared to the synchronous one (Hara et al., 2015; Kokkinara, Slater, \& López-Moliner, 2015; Maselli \& Slater, 2013; Pozeg, Galli, \& Blanke, 2015). Indeed, spatial and spatiotemporal incongruences between the real limb movement and the one that is seen (virtual arm movement) can still lead to high levels of BOI, provided that the fake limb is seen from a 1PP (Kokkinara et al., 2015). In other words, high levels of BOIs could be established even during incongruent multisensory stimulations. Actually, it has been recently suggested that intermodal correspondences are not a necessary prerequisite for the illusion to occur, as long as a spatial congruence between the real and the fake body is provided (Kilteni, Maselli, Kording, \& Slater, 2015).

With the present experiment, we wanted to explore this further: using a virtual reality scenario we investigated whether despite the constant movement of a virtual arm seen from a 1PP, while the real arm is kept immobile, it is still possible to induce the BOI. This will 
contribute to better determine the boundaries of BOIs, and also lead to the development of a possible set-up to be used in clinical settings. In fact, if body ownership could be established under such conditions there could be significant clinical applications, such as with patients with impaired mobility and chronic pain (Adamovich, Fluet, Tunik, \& Merians, 2009; Bagce, Saleh, Adamovich, \& Tunik, 2011; Villiger, Bohli, et al., 2013; Villiger, Estévez, et al., 2013). For instance, it has been shown that the illusory movements of the paralyzed limbs restore motor cortex activity (Giraux \& Sirigu, 2003) and help reducing neuropathic pain in paraplegic patients (Moseley, 2007; Soler et al., 2010). Importantly, no study has, so far, investigated the possible effect of a striking sensorimotor discrepancy between the real and the fake arm (always still VS constantly moving) on BOI. To do this, in the present study we asked our participants to keep their arms still while seeing an avatar's am constantly moving. In two different visual conditions the virtual arm was accompanied or not by synchronous visuo-tactile stimulations. This was done to check whether the presence (or absence) of a synchronous visuo-tactile stimulation could make any difference to the sense of BOI during the visuo-motor sensory mismatch. A third condition involved the use of a virtual purple tube, i.e. a non-corporeal object, in replacement of the virtual arm (see Martini et al., 2014). This condition acted as a baseline, i.e. during this condition no BOI was expected. All conditions were implemented in 1PP.

Since, to our knowledge, no similar studies have been carried so far, we also tested whether distinctive arm/tube movement speeds differently affected the illusion.

\section{Materials and Methods}

\subsection{Participants}

49 participants were initially recruited for the experiment. Two of them were discarded for being ambidextrous and two others for being on psychoactive drugs. Therefore, the final 
sample comprised 45 healthy participants (mean age 25.9, $\mathrm{SD} \pm 5.6,22$ female), randomly assigned to three equally large groups. All participants were right-handed, as evaluated by the revised version of the Edinburgh Handedness Inventory (Veale, 2014). Participants were recruited by advertisement amongst students of the University of East London. Inclusion criteria were normal or normal-to-corrected visionright handedness, and no history of neurological disorders or of drugs use. All participants were naïve regarding the purpose of the experiment and had no previous experience with the virtual or rubber hand illusion. Before the start of the experiment all participants read and signed a written consent form. The experiment had been approved by the local ethics committee and was in accordance with the declaration of Helsinki. Participants did not receive any monetary or academic (students) reward for their participation.

\subsection{Virtual reality system}

The stereoscopic head-mounted display (HMD) was an Oculus Rift DK2 (Oculus VR, Irvine, CA) with a resolution of $960 \times 1080$ per eye and a field of view of $100^{\circ}$, displayed at $60 \mathrm{~Hz}$. The virtual environment was programmed using the Unity platform (Unity Technologies, San Francisco, CA). The tactile feedback (TOUCH group only, see Procedure) was administered via a single small vibrator placed in the middle of the right participant's hand dorsum and controlled via an Arduino board (Arduino LLC, Ivrea, Italy). Noise isolation was ensured by the administration of pink noise via headphones, with a constant volume set at $70 \mathrm{~dB}$ SPL.

\subsection{Questionnaire}

The subjective feeling experienced by the subjects during the exposure to the virtual reality was measured with a questionnaire at the end of each experimental condition. Items were selected from a questionnaire used in seminal work on virtual arm ownership (Slater, Perez- 
Marcos, Ehrsson, \& Sanchez-Vives, 2008). A similar questionnaire was used in another recent study (Zanini, Montalti, Caola, Leadbetter, \& Martini, 2017). The questionnaire items are listed in Table 1

Table 1 - Questionnaire:

\begin{tabular}{|l|l|}
\hline \multicolumn{1}{|c|}{ Label } & Content \\
\hline Q1. Control & $\begin{array}{l}\text { During the experiment there were moments in which I had the } \\
\text { sensation of having more than one right arm. }\end{array}$ \\
\hline Q2. Ownership & $\begin{array}{l}\text { During the experiment there were moments in which I felt as if } \\
\text { the virtual arm/tube was my own arm. }\end{array}$ \\
\hline Q3. Control (Ownership) & $\begin{array}{l}\text { During the experiment there were moments in which I felt as if my } \\
\text { real arm was becoming virtual. }\end{array}$ \\
\hline Q4. Control (Ownership) & $\begin{array}{l}\text { During the experiment there were moments in which the virtual } \\
\text { arm/tube started to look like my own arm in some aspects. }\end{array}$ \\
\hline Q5. Movement & $\begin{array}{l}\text { During the experiment there were moments in which it seemed } \\
\text { that my real arm was moving. }\end{array}$ \\
\hline
\end{tabular}

It should be noted that, due to the different nature of our design and to the uncontrolled constant movement of the virtual arm/tube, only part of the original set of items could be included in the present experiment. For the same reason, we thought that a question about the level of agency ( e.g. "I felt as if I was controlling the movements of the virtual hand") did not make much sense in the present study. The order of the items was randomized for each condition and participant. Importantly, before the administration of the questionnaires, participants were told that there were no right or wrong responses and that the only "right" response was the one that actually corresponded to their feelings. This was done to assure that the data reflected the genuine experience of our participants, hence minimizing the contribution of possible confounding factors such as compliance with "ideal" expected responses. To ensure that each item was correctly interpreted, the questionnaire was read to the participant by the experimenter. The experimenter reading the questions was kept the 
same for all participants. Participants answered verbally using a 7-point Likert scale, with "1" meaning 'totally disagree' and "7" representing 'totally agree'.

Finally, it is important to note that a measure of the proprioceptive drift, i.e. of the subjective shift in the location of the real arm towards the virtual arm, could not be taken in consideration for the current experiment. Indeed, in our case the virtual arm constantly moved back and forth rather than lying still at a fixed distance from the real arm.

\subsection{Procedure}

Upon arrival participants were asked to read and sign the consent form, and to fill in the questionnaires related to the inclusion criteria. Thereafter they sat comfortably on a chair with the left arm resting on the table while the right elbow leaned on a small box, so that the right lower arm and hand were not touching the table. This arrangement was implemented to avoid that the BOI could be reduced by additional sensory mismatch, namely between the expected tactile feedback and the real tactile feedback (i.e. if the right avatar's arm moved left-right on the table the participant would expect a rubbing sensation underneath her/his arm due to friction of the seen virtual arm against the table). Thus, the right avatar's elbow was also rendered on top of a virtual box, so that the right avatar's lower arm and hand moved freely in the air. We used a virtual arm motion on the $x-y$ axis (horizontal), and we did not take in consideration a movement on the $\mathrm{x}-\mathrm{z} / \mathrm{y}-\mathrm{z}$ axis (vertical) because deemed more clearly visible from the participant's perspective. Participants were asked to keep still during the entire VR exposure. They were asked also to keep their right lower arm and hand still so that they could not touch the table. Once the HMD was put on the room's lights were turned off and the pink noise played. The participant's visual scenario was calibrated in order to ensure 
a co-location of the real body with the virtual one. In each condition the participant was asked to explore the virtual room and the virtual body (or tube, depending on the condition). Then participants were asked to keep the focus of attention on the right arm until the end of the virtual exposure. Each virtual condition lasted 120 seconds, much more than the time needed to induce a body ownership illusion with a passive or active movement paradigm (Kalckert \& Ehrsson, 2017).

For the entire duration of the virtual exposure, the experimenters constantly monitored what the participant was looking at in the virtual scenario via a pc screen, and also checked that the real arm remained still.

To avoid diversion of attention from the right arm, the left virtual arm was hidden behind a virtual partition (see Fig.1). Once the participant was ready, the movement of the arm was initiated and kept going until the end of the condition.

A between-subjects design with three different groups was used (see fig.1):

- a TOUCH group where the vision of a virtual ball bouncing on the right avatar's hand dorsum was coupled with a synchronous tactile vibratory stimulus. Every time the ball touched the virtual hand participants felt a tactile stimulus on their right hand dorsum. The virtual ball impacted the virtual hand every 2 seconds and followed the constant movement of the virtual arm, touching it always on the centre of the hand dorsum.

- a NO-TOUCH group where there was only the vision of the virtual arm moving, with no virtual ball displayed and no tactile feedback.

- a TUBE group where there was no virtual ball and no tactile feedback and the virtual arm was replaced by a non-corporeal object, namely a virtual cylinder (tube).

All groups went through three conditions, which only differed with regard to the speed of the arm movement: a "Slow" a "Moderate" and a "Fast" condition, consisting in a constant angular speed $(\omega)$ of $2.5,7.5$ and 12.5 respectively. The lower arm moved $12.5^{\circ}$ towards the 
right and $12.5^{\circ}$ towards the left horizontally, using the elbow as a pivot. The amplitude and the speeds of the movements were selected by the authors in a preliminary pilot. The order of the conditions was counterbalanced among subjects and kept constant for all groups.

After the virtual exposure the HMD was removed and the experimenter read the items of the questionnaire.

\subsection{Data Handling}

Given the nonparametric nature of the data, subjective responses at the questionnaires underwent an aligned rank transform (ART) procedure, as described by Wobbrock and colleagues (Wobbrock, Findlater, Gergle, \& Higgins, 2011). Transformed data were averaged for each item and group, and differences between groups and speeds calculated using mixeddesign 3x3 ANOVAs, run separately for each item, with Speed as a within factor with three levels (Slow, Moderate and Fast) and Group as a between factor with three levels (Touch, No-Touch and Tube). Post-hoc analysis after ANOVAs was conducted with Tukey tests. Statistical comparisons between conditions were conducted with JASP (JASP Team 2016, version 0.7.5.5).

\section{Results}

Table 2 shows the number of subjects, for each group and condition, that agreed with the statements in the questionnaire (i.e. a score $\geq 5$ ). Table 3 reports the averages and standard deviations of raw questionnaire scores per each group and arm/tube movement speed. The two-way ANOVAs on the aligned scores revealed a significant main effect of the factor "Group" for every item of the questionnaire (see fig.2).

Q1: a main effect of the factor "Group" was found $\left(\mathrm{F}_{2,42}=3.31, p=0.046, \eta^{2} \mathrm{p}=0.136\right)$. Since the factor "Group" has more than two levels, a post-hoc comparisons analysis was run to detect which group/s was significantly different from the other/s. Tukey test highlighted a 
strong trend toward significance with a higher feeling of having more than one right arm in the "Touch" group as compared to the "Tube" group ( $p=0.057)$. All other comparisons were not even in trend (all $\left.\mathrm{p}_{\mathrm{s}}>0.1\right)$. Q2: a significant main effect of "Group" was found $\left(\mathrm{F}_{2,42}=\right.$ 3.37, $\left.p=0.033, \eta_{\mathrm{p}}^{2}=0.150\right)$. Post-hoc tests revealed that the group "Touch" reported significantly higher levels of arm ownership as compared to the "Tube" group $(p=0.026)$. Q3: a significant main effect of "Group" was found $\left(\mathrm{F}_{2,42}=5.12, p=0.009, \eta^{2} \mathrm{p}=0.199\right)$. Post-hoc tests revealed that the group "Touch" reported a significantly stronger feeling that the real arm was becoming virtual as compared to the "Tube" group $(p=0.008)$. Also, a trend to significance was found in the comparison "No-Touch" vs "Tube" group, with the former reporting higher levels of this feeling $(p=0.08)$. Q4: a significant main effect of "Group" was found $\left(\mathrm{F}_{2,42}=7.01, p=0.002, \eta_{\mathrm{p}}^{2}=0.250\right)$. Post-hoc tests revealed that both the "Touch" and the "No-Touch" group reported significantly higher levels of the feeling that virtual arm

started to look like their own arm in some aspects, compared to the "Tube" group (respectively $p=0.006$ and $p=0.007$ ). Q5: a significant main effect of "Group" was found $\left(\mathrm{F}_{2,42}=4.11, p=0.023, \eta^{2} \mathrm{p}=0.164\right.$. Post-hoc tests revealed that the group "Touch" reported a significantly stronger illusory sensation that their real arm was moving as compared to the “Tube" group $(p=0.019)$.

No significant main effect of the factor "Speed", nor a significant interaction between the two factors was found for any of the items (all $\mathrm{p}_{\mathrm{s}}>0.05$ ), pointing towards an irrelevant/poor contribution of the arm movement "Speed" factor in shaping the dependent variables taken in consideration in this study.

\section{Discussion}

The main finding of our study is that it is possible to induce a sense of body ownership over a virtual body part despite a dramatic visuo-motor mismatch between the real and the virtual 
limb. Also, we found that an illusory feeling that one's arm is moving while it remains still, can be induced by the vision (in 1PP) of a moving avatar's arm. Both the illusion of ownership and of movement, as indexed by Q1 and Q5, seems to arise clearly only when concomitant synchronous visuo-tactile stimulation is provided. However, given the striking sensory inconsistency between the proprioceptive and the visual realm, we could not expect to induce high levels of BOI in all participants. In fact, even with classical paradigms where perfect visuo-tactile or visuo-motor synchronicity is used, it has been shown how the feeling of ownership over a fake body part occurs in about $75-78 \%$ of the sample, while the other 22 25\% does not experience such illusion (Ehrsson, Holmes, \& Passingham, 2005; Martini et al., 2014). Here we show that with the present paradigm it has been possible to induce the BOI up to the $46.6 \%$ of our sample. Interestingly, in the "No-Touch" group, where no concomitant synchronous visuo-tactile stimulation is provided, the same number of participant (n $7=$ 46.6\%) scored 5 or higher at the Q2 (body ownership) after the slowest arm movement condition. Previous studies using 7-points Likert scales ranging from -3 to +3 , took into account scores of $\geq 1$ to indicate the presence of the illusion (Ehrsson et al., 2004; Kalckert \& Ehrsson, 2012), which is correspondent to our $\geq 5$ criterion in our 1 to 7 Likert scale. This result is quite remarkable, considering that the illusory experience in this group was not supported by a visuo-tactile correspondence. It is noteworthy that the use of a betweensubjects design made the present results free from possible carry-over effects among conditions and, together with the instructions given to participants, kept the level of compliance to ideal expected responses minimal. However, our results also show that a concomitant synchronous visuo-tactile stimulation partly counteracts the disrupting effects of the visuo-motor inconsistency, leading to the overall biggest probability to report the illusion and to feel it stronger. The "Touch" group in particular reported the highest levels of illusion that the real arm was becoming virtual and that the virtual arm resembled the real one $(\mathrm{Q} 3$ 
and Q4). A coupling of these illusory feelings with higher levels of BOI has been found also in previous studies (Perez-Marcos, Sanchez-Vives, \& Slater, 2012; Slater et al., 2008). Despite the fact that items like Q3 and Q4 have been historically deemed as 'control' questions, this idea has been recently challenged in favour of a genuine relationship of these sensations with the feeling of ownership (Perez-Marcos et al., 2012). We back this vision up, supporting the idea that a high level of BOI can sometimes imply illusory feelings that the real and the fake body part start resembling each other. The present study also reveals that coupling the vision of the moving avatar's arm with the visuo-tactile stimulation increases the sensation of having more than one right arm (Q1) as compared to the vision of a moving noncorporeal object (i.e. the tube). In a similar experiment it was found that the same question was far from being significantly different from chance after visuo-tactile stimulation (Slater et al., 2008). Nevertheless, it has been shown that it is possible to induce a strong illusion of ownership over multiple limbs synchronously stroking two seen prosthetic hands while the real hand is hidden from the view (Ehrsson, 2009), or synchronously stroking a prosthetic hand and the real hand both kept in view (Guterstam, Petkova, \& Ehrsson, 2011). Our paradigm implied a key difference with the aforementioned studies, namely we introduced a constant visuo-motor mismatch. We should also point out that in our experiment, the sensation of having more than one right arm has been reported the least amount of times by participants in both the "Touch" and "No-Touch" group compared to the other illusions. This, coupled with the non-significant $\mathrm{p}$-value after the ANOVA on the transformed values, make it hard for us to draw any conclusions about the role of looking at a moving arm on Q1. After all, a questionnaire item asking about this sensation classically deemed to be a control question (Botvinick \& Cohen, 1998).

Our results show that it is possible to induce an illusory sensation that one's own real arm is moving despite the fact that it is completely still. This illusion seems likely to take place only 
with the concomitant aid of a congruent visuo-tactile stimulation, although if we look at the percentages of the participants that scored at least "5" at Q5, we have a similar number of participants reporting the illusion between the "Touch" and the "No-Touch" group. Kinesthetic illusions have been reported during tendon vibration (e.g. Fusco et al., 2016; Jones, 1988; Naito et al., 2002; Tidoni et al., 2015) or while viewing videos of moving limbs superimposed to one's real limb (Aoyama, Kaneko, Hayami, \& Shibata, 2012; Kaneko, Yasojima, \& Kizuka, 2007; Kaneko, Inada, Matsuda, Shibata, \& Koyama, 2016) not to mention during mirror therapy interventions (Ramachandran \& Rogers-Ramachandran, 1996). The possibility to induce illusory movements could be beneficial in numerous cases. For instance it has been shown how the illusory movement of one's limb can prevent the disruption of sensorimotor areas usually occurring during immobilization (Roll et al., 2012), or even restore motor cortex activity in patients with brachial plexus avulsion (Giraux \& Sirigu, 2003). Further applications of kinesthetic illusions can be found in the treatment of patients affected by complex-regional pain syndrome (Gay et al., 2007), or those suffering from post-operative acute pain (Imai, Osumi, \& Morioka, 2016). In the case of pain, these beneficial effects might be boosted in future studies by the vision of one's own body (Longo, Betti, Aglioti, \& Haggard, 2009; M. Martini et al., 2014; M.Martini, 2016) and/or by the highly distractive power of virtual reality scenarios (Hoffman et al., 2011; Malloy \& Milling, 2010). Virtual reality in particular has been identified as a method which, due to its versatility, has a great advantage over classical method like mirror therapy (Ramachandran \& Altschuler, 2009) and it is being successfully used both in neuroscientific research and in therapeutical approaches (see Bohil et al., 2011 for a review).

As a methodological note, we are aware that some RHI/VHI studies have made use of physiological measures, like skin conductance responses (SCR) and/or heart rate (HR) as 'objective measures' of body ownership. However, an increase of SCR has been reported not 
only during the vision of one's body but also during the observation of another's body part in pain (Avenanti, Sirigu, \& Aglioti, 2010; De Coster, Verschuere, Goubert, Tsakiris, \& Brass, 2013; Fusaro, Tieri, \& Aglioti, 2016; Pfabigan et al., 2015). Similarly, reduction of HR has been found in response to either pain-inducing or pleasant stimuli applied to either "one's own" virtual body or another's body (Fusaro et al., 2016). Therefore, these evidences make the reliance on such 'objective' measures of body ownership more problematic as they do not seem to distinguish between 'my body' and 'another's body'.

Moreover, in a recent study by Ma \& Hommel (Ma \& Hommel, 2015), it was found that SCR values were generally higher when a threat was applied on a virtual hand than on a virtual rectangle, but the authors also found that this effect did not interact with the type of visuotactile stimulation (synchronous-asynchronous) meant to modulate body ownership. This is in line with previous findings from the same authors (Ma \& Hommel, 2013), who observed increases in SCR levels in response to a threat targeting a virtual hand that they did not perceive as their own. Thus, again, this 'objective measure' of body ownership can, in fact, reflect an autonomic response that is not dependent on body ownership, or at least not only on it. This said, despite our methodological precautions, we still cannot completely rule out the possible contribution of demand characteristics (Orne, 1969; Orne, 1962), so other studies are needed to confirm the current findings.

\section{Conclusions}

The present study shows that it is possible to induce a sense of body ownership over a fake limb even under striking visuo-motor discrepancies between the virtual and the real arm. However, the percentages of the participants that experienced the illusion in the present study are lower than the ones generally reported under classical bodily illusions paradigms. It is also worthy of note that, when considering different arm speeds, the amount of participants 
that experience the illusion of BOI and of arm movement does not follow a linear trend, and it is different for each group. Nonetheless, our findings show that the slowest movement maximizes the chances of experiencing the BOI in both groups where the avatar's arm is displayed. Still, the current study has to be considered a preliminary work and the results need future confirmation. Future studies could investigate whether the different limb movements' amplitudes, speeds or types can determine higher levels of body ownership and kinesthetic illusion to those reported in the present work.

\section{Acknowledgements}

The authors want to thank Diana Chiari and Keighley Courtney Priest for their help with the data collection.

The research was conducted in the absence of any commercial or financial relationships that could be construed as a potential conflict of interest.

\section{Bibliography}

Adamovich, S. V, Fluet, G. G., Tunik, E., \& Merians, A. S. (2009). Sensorimotor training in virtual reality: a review. NeuroRehabilitation, 25(1), 29-44. http://doi.org/10.3233/NRE-2009-0497

Aoyama, T., Kaneko, F., Hayami, T., \& Shibata, E. (2012). The effects of kinesthetic illusory 
sensation induced by a visual stimulus on the corticomotor excitability of the leg muscles. Neuroscience Letters (Vol. 514).

Avenanti, A., Sirigu, A., \& Aglioti, S. M. (2010). Racial Bias Reduces Empathic Sensorimotor Resonance with Other-Race Pain. Current Biology, 20(11), 1018-1022. http://doi.org/10.1016/j.cub.2010.03.071

Bagce, H. F., Saleh, S., Adamovich, S. V, \& Tunik, E. (2011). Visuomotor discordance in virtual reality: effects on online motor control. Conference Proceedings : ... Annual International Conference of the IEEE Engineering in Medicine and Biology Society. IEEE Engineering in Medicine and Biology Society. Conference, 2011, 7262-5. http://doi.org/10.1109/IEMBS.2011.6091835

Banakou, D., Groten, R., \& Slater, M. (2013). Illusory ownership of a virtual child body causes overestimation of object sizes and implicit attitude changes. Proceedings of the National Academy of Sciences of the United States of America, 110(31), 12846-51. http://doi.org/10.1073/pnas.1306779110

Bergström, I., Kilteni, K., \& Slater, M. (2016). First-Person Perspective Virtual Body Posture Influences Stress: A Virtual Reality Body Ownership Study. PloS One, 11(2), e0148060. http://doi.org/10.1371/journal.pone.0148060

Berlucchi, G., \& Aglioti, S. M. (2010). The body in the brain revisited. Experimental Brain Research, 200(1), 25-35. http://doi.org/10.1007/s00221-009-1970-7

Bohil, C. J., Alicea, B., \& Biocca, F. a. (2011). Virtual reality in neuroscience research and therapy. Nature Reviews. Neuroscience, 12(12), 752-62. http://doi.org/10.1038/nrn3122

Botvinick, M., \& Cohen, J. (1998). Rubber hands "feel" touch that eyes see. Nature, 391(6669), 756. http://doi.org/10.1038/35784 
Crucianelli, L., Krahé, C., Jenkinson, P. M., \& Fotopoulou, A. (2017). Interoceptive ingredients of body ownership: Affective touch and cardiac awareness in the rubber hand illusion. Cortex. http://doi.org/10.1016/j.cortex.2017.04.018

De Coster, L., Verschuere, B., Goubert, L., Tsakiris, M., \& Brass, M. (2013). I suffer more from your pain when you act like me: being imitated enhances affective responses to seeing someone else in pain. Cognitive, Affective \& Behavioral Neuroscience, 13(3), 519-32. http://doi.org/10.3758/s13415-013-0168-4

Ehrsson, H. H. (2009). How many arms make a pair? Perceptual illusion of having an additional limb. Perception, 38(2), 310-2. Retrieved from http://www.ncbi.nlm.nih.gov/pubmed/19400438

Ehrsson, H. H., Holmes, N. P., \& Passingham, R. E. (2005). Touching a Rubber Hand: Feeling of Body Ownership Is Associated with Activity in Multisensory Brain Areas. Journal of Neuroscience, 25(45), 10564-10573. http://doi.org/10.1523/JNEUROSCI.0800-05.2005

Ehrsson, H. H., Spence, C., \& Passingham, R. E. (2004). That's my hand! Activity in premotor cortex reflects feeling of ownership of a limb. Science (New York, N.Y.), 305(5685), 875-7. http://doi.org/10.1126/science.1097011

Feinberg, T. E., Venneri, A., Simone, A. M., Fan, Y., \& Northoff, G. (2010). The neuroanatomy of asomatognosia and somatoparaphrenia. Journal of Neurology, Neurosurgery, and Psychiatry, 81(3), 276-81. http://doi.org/10.1136/jnnp.2009.188946

Fusaro, M., Tieri, G., \& Aglioti, S. M. (2016). Seeing pain and pleasure on self and others: behavioral and psychophysiological reactivity in immersive virtual reality. Journal of Neurophysiology, 116(6), 2656-2662. http://doi.org/10.1152/jn.00489.2016 
Fusco, G., Tidoni, E., Barone, N., Pilati, C., \& Aglioti, S. M. (2016). Illusion of arm movement evoked by tendon vibration in patients with spinal cord injury. Restorative Neurology and Neuroscience. http://doi.org/10.3233/RNN-160660

Gay, A., Parratte, S., Salazard, B., Guinard, D., Pham, T., Legré, R., \& Roll, J. P. (2007). Proprioceptive feedback enhancement induced by vibratory stimulation in complex regional pain syndrome type I: an open comparative pilot study in 11 patients. Joint, Bone, Spine : Revue Du Rhumatisme, 74(5), 461-6.

http://doi.org/10.1016/j.jbspin.2006.10.010

Giraux, P., \& Sirigu, A. (2003). Illusory movements of the paralyzed limb restore motor cortex activity. NeuroImage, 20, S107-S111. http://doi.org/10.1016/j.neuroimage.2003.09.024

Guterstam, A., Abdulkarim, Z., \& Ehrsson, H. H. (2015). Illusory ownership of an invisible body reduces autonomic and subjective social anxiety responses. Scientific Reports, 5, 9831. http://doi.org/10.1038/srep09831

Guterstam, A., Gentile, G., \& Ehrsson, H. H. (2013). The invisible hand illusion: multisensory integration leads to the embodiment of a discrete volume of empty space. Journal of Cognitive Neuroscience, 25(7), 1078-99. http://doi.org/10.1162/jocn_a_00393

Guterstam, A., Petkova, V. I., \& Ehrsson, H. H. (2011). The illusion of owning a third arm. PloS One, 6(2), e17208. http://doi.org/10.1371/journal.pone.0017208

Haggard, P., \& Jundi, S. (2009). Rubber hand illusions and size-weight illusions: selfrepresentation modulates representation of external objects. Perception, 38(12), 1796803. Retrieved from http://www.ncbi.nlm.nih.gov/pubmed/20192129 
Hara, M., Pozeg, P., Rognini, G., Higuchi, T., Fukuhara, K., Yamamoto, A., ... Salomon, R. (2015). Voluntary self-touch increases body ownership. Frontiers in Psychology, 6, 1509. http://doi.org/10.3389/fpsyg.2015.01509

Hoffman, H. G., Chambers, G. T., Meyer, W. J. 3rd, Arceneaux, L. L., Russell, W. J., Seibel, E. J., ... Patterson, D. R. (2011). Virtual reality as an adjunctive non-pharmacologic analgesic for acute burn pain during medical procedures. Annals of Behavioral Medicine: A Publication of the Society of Behavioral Medicine, 41(2), 183-191. http://doi.org/10.1007/s12160-010-9248-7

Imai, R., Osumi, M., \& Morioka, S. (2016). Influence of illusory kinesthesia by vibratory tendon stimulation on acute pain after surgery for distal radius fractures: a quasirandomized controlled study. Clinical Rehabilitation, 30(6), 594-603. http://doi.org/10.1177/0269215515593610

Jones, L. A. (1988). Motor illusions: what do they reveal about proprioception? Psychological Bulletin, 103(1), 72-86. Retrieved from http://www.ncbi.nlm.nih.gov/pubmed/3279446

Kalckert, A., \& Ehrsson, H. H. (2012). Moving a Rubber Hand that Feels Like Your Own: A Dissociation of Ownership and Agency. Frontiers in Human Neuroscience, 6. http://doi.org/10.3389/fnhum.2012.00040

Kalckert, A., \& Ehrsson, H. H. (2017). The Onset Time of the Ownership Sensation in the Moving Rubber Hand Illusion. Frontiers in Psychology, 8, 344. http://doi.org/10.3389/fpsyg.2017.00344

Kaneko, F., Inada, T., Matsuda, N., Shibata, E., \& Koyama, S. (2016). Acute Effect of Visually Induced Kinesthetic Illusion in Patients with Stroke: A Preliminary Report. International Journal of Neurorehabilitation, 3(3), 1-6. http://doi.org/10.4172/2376- 
0281.1000212

Kaneko, F., Yasojima, T., \& Kizuka, T. (2007). Kinesthetic illusory feeling induced by a finger movement movie effects on corticomotor excitability. Neuroscience, 149(4), 97684. http://doi.org/10.1016/j.neuroscience.2007.07.028

Kilteni, K., Maselli, A., Kording, K. P., \& Slater, M. (2015). Over my fake body: body ownership illusions for studying the multisensory basis of own-body perception. Frontiers in Human Neuroscience, 9, 141. http://doi.org/10.3389/fnhum.2015.00141

Kilteni, K., Normand, J.-M., Sanchez-Vives, M. V, \& Slater, M. (2012). Extending body space in immersive virtual reality: a very long arm illusion. PloS One, 7(7), e40867. http://doi.org/10.1371/journal.pone.0040867

Kokkinara, E., Slater, M., \& López-Moliner, J. (2015). The Effects of Visuomotor Calibration to the Perceived Space and Body, through Embodiment in Immersive Virtual Reality. ACM Transactions on Applied Perception, 13(1), 1-22. http://doi.org/10.1145/2818998

Longo, M. R., Betti, V., Aglioti, S. M., \& Haggard, P. (2009). Visually induced analgesia: seeing the body reduces pain. The Journal of Neuroscience: The Official Journal of the Society for Neuroscience, 29(39), 12125-12130.

http://doi.org/10.1523/JNEUROSCI.3072-09.2009

Ma, K., \& Hommel, B. (2013). The virtual-hand illusion: effects of impact and threat on perceived ownership and affective resonance. Frontiers in Psychology, 4. http://doi.org/10.3389/fpsyg.2013.00604

Ma, K., \& Hommel, B. (2015). The role of agency for perceived ownership in the virtual hand illusion. Consciousness and Cognition, 36, 277-288. 
http://doi.org/10.1016/j.concog.2015.07.008

Ma, K., Sellaro, R., Lippelt, D. P., \& Hommel, B. (2016). Mood migration: How enfacing a smile makes you happier. Cognition, 151, 52-62. http://doi.org/10.1016/j.cognition.2016.02.018

Maister, L., Slater, M., Sanchez-Vives, M. V, Tsakiris, M., Baumeister, R. F., Bushman, B. J., ... al., et. (2015). Changing bodies changes minds: owning another body affects social cognition. Trends in Cognitive Sciences, 19(1), 6-12. http://doi.org/10.1016/j.tics.2014.11.001

Makin, T. R., Holmes, N. P., \& Ehrsson, H. H. (2008). On the other hand: Dummy hands and peripersonal space. Behavioural Brain Research, 191(1), 1-10. http://doi.org/10.1016/j.bbr.2008.02.041

Malloy, K. M., \& Milling, L. S. (2010). The effectiveness of virtual reality distraction for pain reduction: A systematic review. Clinical Psychology Review, 30(8), 1011-1018. http://doi.org/10.1016/j.cpr.2010.07.001

Martinaud, O., Besharati, S., Jenkinson, P. M., \& Fotopoulou, A. (2017). Ownership illusions in patients with body delusions: Different neural profiles of visual capture and disownership. Cortex, 87, 174-185. http://doi.org/10.1016/j.cortex.2016.09.025

Martini, M. (2016). Real, rubber or virtual: The vision of “one's own” body as a means for pain modulation. A narrative review. Consciousness and Cognition, 43, 143-51. http://doi.org/10.1016/j.concog.2016.06.005

Martini, M., Kilteni, K., Maselli, A., \& Sanchez-Vives, M. V. (2015). The body fades away: investigating the effects of transparency of an embodied virtual body on pain threshold and body ownership. Scientific Reports, 5, 13948. http://doi.org/10.1038/srep13948 
Martini, M., Perez-Marcos, D., \& Sanchez-Vives, M. V. (2013). What Color is My Arm? Changes in Skin Color of an Embodied Virtual Arm Modulates Pain Threshold. Frontiers in Human Neuroscience, 7(July), 438. http://doi.org/10.3389/fnhum.2013.00438

Martini, M., Perez-Marcos, D., \& Sanchez-Vives, M. V. (2014). Modulation of pain threshold by virtual body ownership. European Journal of Pain (London, England). http://doi.org/10.1002/j.1532-2149.2014.00451.x

Maselli, A., \& Slater, M. (2013). The building blocks of the full body ownership illusion. Frontiers in Human Neuroscience, 7, 83. http://doi.org/10.3389/fnhum.2013.00083

Moseley, L. G. (2007). Using visual illusion to reduce at-level neuropathic pain in paraplegia. Pain, 130(3), 294-298. http://doi.org/10.1016/j.pain.2007.01.007

Naito, E., Roland, P. E., \& Ehrsson, H. H. (2002). I Feel My Hand Moving: A New Role of the Primary Motor Cortex in Somatic Perception of Limb Movement. Neuron, 36(5), 979-988. http://doi.org/10.1016/S0896-6273(02)00980-7

Orne, M. T. (1962). On the social psychology of the psychological experiment: With particular reference to demand characteristics and their implications. American Psychologist, 17(11), 776-783. http://doi.org/10.1037/h0043424

Orne, M. T. (1969). Demand characteristics and the concept of quasi-controls. In R. Rosenthal \& R. Rosnow (Ed.), Artifact in behavioral research. (pp. 143-179). New York: Academic Press.

Osimo, S. A., Pizarro, R., Spanlang, B., \& Slater, M. (2015). Conversations between self and self as Sigmund Freud--A virtual body ownership paradigm for self counselling. Scientific Reports, 5, 13899. http://doi.org/10.1038/srep13899 
Osumi, M., Imai, R., Ueta, K., Nobusako, S., \& Morioka, S. (2014). Negative body image associated with changes in the visual body appearance increases pain perception. PloS One, 9(9), e107376. http://doi.org/10.1371/journal.pone.0107376

Peck, T. C., Seinfeld, S., Aglioti, S. M., \& Slater, M. (2013). Putting yourself in the skin of a black avatar reduces implicit racial bias. Consciousness and Cognition, 22(3), 779-87. http://doi.org/10.1016/j.concog.2013.04.016

Perez-Marcos, D., Sanchez-Vives, M. V, \& Slater, M. (2012). Is my hand connected to my body? The impact of body continuity and arm alignment on the virtual hand illusion. Cognitive Neurodynamics, 6(4), 295-305. http://doi.org/10.1007/s11571-011-9178-5

Petkova, V. I., \& Ehrsson, H. H. (2008). If I were you: perceptual illusion of body swapping. PloS One, 3(12), e3832. http://doi.org/10.1371/journal.pone.0003832

Petkova, V. I., Khoshnevis, M., \& Ehrsson, H. H. (2011). The perspective matters! Multisensory integration in ego-centric reference frames determines full-body ownership. Frontiers in Psychology, 2, 35. http://doi.org/10.3389/fpsyg.2011.00035

Pfabigan, D. M., Seidel, E.-M., Wucherer, A. M., Keckeis, K., Derntl, B., \& Lamm, C. (2015). Affective empathy differs in male violent offenders with high- and low-trait psychopathy. Journal of Personality Disorders, 29(1), 42-61. http://doi.org/10.1521/pedi_2014_28_145

Pozeg, P., Galli, G., \& Blanke, O. (2015). Those are Your Legs: The Effect of Visuo-Spatial Viewpoint on Visuo-Tactile Integration and Body Ownership. Frontiers in Psychology, 6, 1749. http://doi.org/10.3389/fpsyg.2015.01749

Preston, C., \& Ehrsson, H. H. (2016). Illusory Obesity Triggers Body Dissatisfaction Responses in the Insula and Anterior Cingulate Cortex. Cerebral Cortex (New York, 
N.Y. : 1991), 26(12), 4450-4460. http://doi.org/10.1093/cercor/bhw313

Ramachandran, V. S., \& Altschuler, E. L. (2009). The use of visual feedback, in particular mirror visual feedback, in restoring brain function. Brain : A Journal of Neurology, 132(Pt 7), 1693-710. http://doi.org/10.1093/brain/awp135

Ramachandran, V. S., \& Rogers-Ramachandran, D. (1996). Synaesthesia in Phantom Limbs Induced with Mirrors. Proceedings of the Royal Society B: Biological Sciences, 263(1369), 377-386. http://doi.org/10.1098/rspb.1996.0058

Roll, R., Kavounoudias, A., Albert, F., Legré, R., Gay, A., Fabre, B., \& Roll, J. P. (2012). Illusory movements prevent cortical disruption caused by immobilization. NeuroImage, 62(1), 510-9. http://doi.org/10.1016/j.neuroimage.2012.05.016

Slater, M., Perez-Marcos, D., Ehrsson, H. H., \& Sanchez-Vives, M. V. (2008). Towards a digital body: the virtual arm illusion. Frontiers in Human Neuroscience, 2, 6. http://doi.org/10.3389/neuro.09.006.2008

Slater, M., Spanlang, B., Sanchez-Vives, M. V, \& Blanke, O. (2010). First person experience of body transfer in virtual reality. PloS One, 5(5), e10564. http://doi.org/10.1371/journal.pone.0010564

Soler, M. D., Kumru, H., Pelayo, R., Vidal, J., Tormos, J. M., Fregni, F., ... Pascual-Leone, A. (2010). Effectiveness of transcranial direct current stimulation and visual illusion on neuropathic pain in spinal cord injury. Brain: A Journal of Neurology, 133(9), 25652577. http://doi.org/10.1093/brain/awq184

Tidoni, E., Fusco, G., Leonardis, D., Frisoli, A., Bergamasco, M., \& Aglioti, S. M. (2015). Illusory movements induced by tendon vibration in right- and left-handed people. Experimental Brain Research, 233(2), 375-83. http://doi.org/10.1007/s00221-014-4121- 
Tieri, G., Tidoni, E., Pavone, E. F., \& Aglioti, S. M. (2015). Mere observation of body discontinuity affects perceived ownership and vicarious agency over a virtual hand. Experimental Brain Research, 233(4), 1247-59. http://doi.org/10.1007/s00221-015$4202-3$

Tsakiris, M., Carpenter, L., James, D., \& Fotopoulou, A. (2010). Hands only illusion: multisensory integration elicits sense of ownership for body parts but not for noncorporeal objects. Experimental Brain Research, 204(3), 343-352. http://doi.org/10.1007/s00221-009-2039-3

Tsakiris, M., \& Haggard, P. (2005). The rubber hand illusion revisited: visuotactile integration and self-attribution. Journal of Experimental Psychology. Human Perception and Performance, 31(1), 80-91. http://doi.org/10.1037/0096-1523.31.1.80

Vallar, G., \& Ronchi, R. (2009). Somatoparaphrenia: a body delusion. A review of the neuropsychological literature. Experimental Brain Research, 192(3), 533-551. http://doi.org/10.1007/s00221-008-1562-y

van der Hoort, B., \& Ehrsson, H. H. (2014). Body ownership affects visual perception of object size by rescaling the visual representation of external space. Attention, Perception \& Psychophysics, 76(5), 1414-28. http://doi.org/10.3758/s13414-014-0664-9

van der Hoort, B., Guterstam, A., \& Ehrsson, H. H. (2011). Being Barbie: the size of one's own body determines the perceived size of the world. PloS One, 6(5), e20195. http://doi.org/10.1371/journal.pone.0020195

Veale, J. F. (2014). Edinburgh Handedness Inventory - Short Form: a revised version based on confirmatory factor analysis. Laterality, 19(2), 164-77. 
http://doi.org/10.1080/1357650X.2013.783045

Villiger, M., Bohli, D., Kiper, D., Pyk, P., Spillmann, J., Meilick, B., ... Eng, K. (2013). Virtual reality-augmented neurorehabilitation improves motor function and reduces neuropathic pain in patients with incomplete spinal cord injury. Neurorehabilitation and Neural Repair, 27(8), 675-83. http://doi.org/10.1177/1545968313490999

Villiger, M., Estévez, N., Hepp-Reymond, M.-C., Kiper, D., Kollias, S. S., Eng, K., \& HotzBoendermaker, S. (2013). Enhanced activation of motor execution networks using action observation combined with imagination of lower limb movements. PloS One, 8(8), e72403. http://doi.org/10.1371/journal.pone.0072403

Wobbrock, J. O., Findlater, L., Gergle, D., \& Higgins, J. J. (2011). The aligned rank transform for nonparametric factorial analyses using only anova procedures. In Proceedings of the 2011 annual conference on Human factors in computing systems CHI '11 (p. 143). New York, New York, USA: ACM Press. http://doi.org/10.1145/1978942.1978963

Zanini, A., Montalti, M., Caola, B., Leadbetter, A., \& Martini, M. (2017). Pain during illusory own arm movement: a study in immersive virtual reality. European Medical Journal, 2(2), 90-97.

\section{Figure legends}

Fig.1 
From left to right: an illustrative picture of participant and three screenshots of the three different visual scenarios (groups) in VR. From the left: "Touch", "No-Touch" and "Tube" group.

Fig.2

Mean and standard error of the aligned rank transform (ART) index reported by the three different groups for the only significant effect found in the ANOVA (main effect of Group). Asterisks indicate significant comparisons $(* \mathrm{p}<0.05 ; * * \mathrm{p}<0.01)$.

\begin{tabular}{|c|c|c|c|c|c|c|}
\hline & & Q1 & Q2 & Q3 & $\mathrm{Q}^{4}$ & Q5 \\
\hline \multirow{4}{*}{ Touch } & Slow & 0 & $7(46.6 \%)$ & $5(33.3 \%)$ & $4(26.6 \%)$ & $7(46.6 \%)$ \\
\hline & Moderate & $5(33.3 \%)$ & $7(46.6 \%)$ & $6(40 \%)$ & $6(40 \%)$ & $9(60 \%)$ \\
\hline & Fast & $1(6.6 \%)$ & $6(40 \%)$ & $5(33.3 \%)$ & $3(20 \%)$ & $7(46.6 \%)$ \\
\hline & TOTAL & $6(13.3 \%)$ & $20(44 \%)$ & $\begin{array}{c}16 \\
(35.5 \%)\end{array}$ & $\begin{array}{c}13 \\
(28.8 \%)\end{array}$ & $\begin{array}{c}23 \\
(51.1 \%)\end{array}$ \\
\hline \multirow{5}{*}{ No-Touch } & Slow & $3(20 \%)$ & $7(46.6 \%)$ & $3(20 \%)$ & $5(33.3 \%)$ & $7(46.6 \%)$ \\
\hline & Moderate & $3(20 \%)$ & $3(20 \%)$ & $3(20 \%)$ & $6(40 \%)$ & $7(46.6 \%)$ \\
\hline & Fast & $1(6.6 \%)$ & $5(33.3 \%)$ & $3(20 \%)$ & $7(46.6 \%)$ & $8(53.3 \%)$ \\
\hline & TOTAL & $7(15.5 \%)$ & $\begin{array}{c}15 \\
(33.3 \%)\end{array}$ & $9(20 \%)$ & $18(40 \%)$ & $\begin{array}{c}22 \\
(48.8 \%)\end{array}$ \\
\hline & Slow & $1(6.6 \%)$ & $2(13.3 \%)$ & $1(6.6 \%)$ & $1(6.6 \%)$ & $2(13.3 \%)$ \\
\hline
\end{tabular}




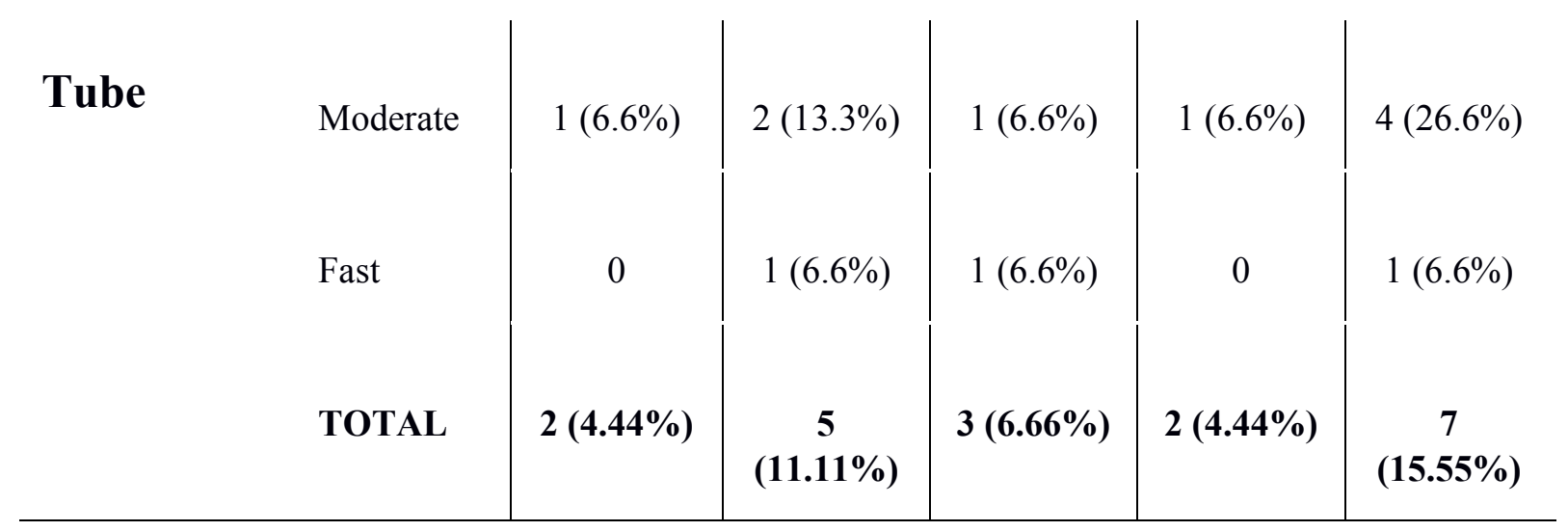

\section{Table 2}

Number (and percentage) of participants that indicated the presence of the illusion, i.e. a scored 5 or higher per each question, according to each group and arm/tube movement speed.

\begin{tabular}{|c|c|c|c|c|c|c|}
\hline & & Q1 & $\mathrm{Q}^{2}$ & Q3 & $\mathrm{Q}^{4}$ & Q5 \\
\hline \multirow{3}{*}{ Touch } & Slow & $1,67(1,05)$ & $4,00(2,24)$ & $3,40(1,84)$ & $3,27(1,75)$ & $4,00(2,30)$ \\
\hline & Moderate & $2,93(2,15)$ & $4,40(2,29)$ & $3,87(2,03)$ & $3,67(2,09)$ & $4,67(2,26)$ \\
\hline & Fast & $1,73(1,28)$ & $3,87(1,85)$ & $3,47(1,92)$ & $3,00(1,56)$ & $3,93(1,98)$ \\
\hline \multirow{3}{*}{ No-Touch } & Slow & $2,27(1,83)$ & $3,40(1,88)$ & $2,47(1,60)$ & $3,13(1,92)$ & $3,60(2,26)$ \\
\hline & Moderate & $2,33(1,88)$ & $3,07(1,79)$ & $2,53(1,96)$ & $3,53(1,92)$ & $3,47(2,23)$ \\
\hline & Fast & $2,27(1,28)$ & $2,93(2,05)$ & $2,67(1,68)$ & $3,67(2,16)$ & $4,07(2,58)$ \\
\hline
\end{tabular}




\begin{tabular}{|c|c|c|c|c|c|c|}
\hline & Slow & $1,67(1,18)$ & $2,80(1,32)$ & $2,27(1,39)$ & $1,73(1,22)$ & $2,60(1,64)$ \\
\hline Tube & Moderate & $1,60(1,12)$ & $2,47(1,55)$ & $2,07(1,33)$ & $1,80(1,21)$ & $2,53(1,92)$ \\
\hline & Fast & $1,33(0,82)$ & $2,07(1,39)$ & $1,60(1,12)$ & $1,60(0,91)$ & $2,27(1,49)$ \\
\hline
\end{tabular}

\section{Table 3}

Averages (and SD) of raw questionnaire scores per each group and arm/tube movement speed. 